\title{
Asymptotic behavior of the number of Eulerian orientations of graphs
}

\author{
Mikhail Isaev
}

\begin{abstract}
We consider the class of simple graphs with large algebraic connectivity (the second-smallest eigenvalue of the Laplacian matrix). For this class of graphs we determine the asymptotic behavior of the number of Eulerian orientations. In addition, we establish some new properties of the Laplacian matrix, as well as an estimate of a conditionality of matrices with the asymptotic diagonal predominance
\end{abstract}

\section{Introduction}

The eulerian orientation of the graph $G$ is the orientation of its edges such that for every vertex the number of incoming edges and outgoing edges are equal. We denote the number of Eulerian orientations $E O(G)$. It is easy clear that $E O(G)=0$, if the degree of at least one vertex of $G$ is odd. Eulerian orientations of the complete graph $K_{n}$ are called regular tournaments.

In [9] it is shown that for even $n \rightarrow \infty$

$$
E O\left(K_{n}\right)=\left(\frac{2^{n+1}}{\pi n}\right)^{(n-1) / 2} n^{1 / 2} e^{-1 / 2}\left(1+O\left(n^{-1 / 2+\varepsilon}\right)\right)
$$

for any $\varepsilon>0$.

Undirected graphs without loops and multiple edges are called simple.

The problem of counting the number of the Eulerian orienations of an undirected simple graph is complete for the class \#P, see [6]. Thus this problem is difficult in terms of complexity theory.

In addition, for the case of loopless $2 d$-regular graph $G$ with $n$ vertices the following estimates hold, see [4, [10]:

$$
2^{d}\left(\frac{(2 d-1) ! !}{d !}\right)^{n-1} \leq E O(G) \leq\left(\frac{(2 d) !}{d ! \cdot d !}\right)^{n / 2}
$$

An improvement of the upper bound for the regular graph case and some additional studies in this direction were fulfilled in 5 .

For the simple graph $G$ we define $n \times n$ matrix $Q$ by

$$
Q_{j k}=\left\{\begin{aligned}
-1, & \left\{v_{j}, v_{k}\right\} \in E G, \\
d_{j}, & j=k \\
0, & \text { otherwise }
\end{aligned}\right.
$$

where $n=|V G|$ and $d_{j}$ denotes the degree of $v_{j} \in V G$. The matrix $Q=Q(G)$ is called the Laplacian matrix of the graph $G$. The eigenvalues $\lambda_{1} \leq \lambda_{2} \leq \ldots \leq \lambda_{n}$ 
of the matrix $Q$ are always non-negative real numbers and the number of zero eigenvalues of $Q$ coincides with the number of connected components of $G$, in particular, $\lambda_{1}=0$. The eigenvalue $\lambda_{2}$ is called the algebraic connectivity of the graph $G$. (For more information about the spectral properties of the Laplace matrix see, for example, [2] and [7.)

According to the Kirchhoffs Matrix-Tree-Theorem, see 3, we have that

$$
t(G)=\frac{1}{n} \lambda_{2} \lambda_{3} \cdots \lambda_{n},
$$

where $t(G)$ denotes the number of spanning trees of the graph $G$.

In the present work we generalize approach of $[9$. We determine the asymptotic behavior of the number of Eulerian orientations of simple graphs with large algebraic connectivity, see Theorem 2.1. In Section 2 we give conventions and notations and formulate the main result.

In Section 3 we prove some basic properties of the Laplacian matrix. Some statements seem to be of independent interest, for example, we obtain an estimate of a conditionality of matrices with the asymptotic diagonal predominance, see Lemma 3.2 Also, we prove the following property of simple graphs with large algebraic connectivity: when you remove the vertex the number of spanning trees decreases by no more than $c n$ times, for some $c>0$ depending only on $\lambda_{2} / n$, see. Corollary 3.3 .

We prove the main result in Section 4. We express $E O(G)$ in terms of an $n$-dimensional integral using Cauchys formula. The derivation of asymptotic estimation of this integral uses three lemmas (Lemma 4.1, 4.2, 4.3), whose proofs are given in Section 5 .

\section{Main result}

Let $p \geq 1$ be a real number and $\vec{x} \in \mathbb{R}^{n}$. We use notation

$$
\|\vec{x}\|_{p}=\left(\sum_{j=1}^{n}\left|x_{j}\right|^{p}\right)^{1 / p} .
$$

For $p=\infty$ we have the maximum norm

$$
\|\vec{x}\|_{\infty}=\max _{j}\left|x_{j}\right| .
$$

The matrix norm corresponding to the $p$-norm for vectors is

$$
\|A\|_{p}=\sup _{\vec{x} \neq 0} \frac{\|A \vec{x}\|_{p}}{\|\vec{x}\|_{p}} .
$$

If $A$ is the matrix of self-adjoint operator (symmetric matrix) then the norm $\|A\|_{2}$ is equal to the largest module of eigenvalue of $A$ and the following inequality holds:

$$
\|A\|_{p} \geq\|A\|_{2} .
$$

For invertible matrices one can define the condition number.

$$
\mu_{p}(A)=\|A\|_{p} \cdot\left\|A^{-1}\right\|_{p} \geq\left\|A A^{-1}\right\|_{p}=1 .
$$


If $f$ is bounded both above and below by $g$ asymptotically, we use the notation

$$
f(n)=\Theta_{k_{1}, k_{2}}(g(n)),
$$

which implies as $n \rightarrow \infty$, eventually

$$
k_{1}|g(n)| \leq|f(n)| \leq k_{2}|g(n)| .
$$

When functions $f$ and $g$ depend not only on $n$, but also on other parameters $\vec{\xi}$, we use notation (2.6) meaning that condition (2.7) holds uniformly for all possible values of $\vec{\xi}$.

The main result of the present work is the following theorem.

Theorem 2.1. Let $G$ be simple graph with $n$ vertices having even degree and the algebraic connectivity $\lambda_{2} \geq \gamma n$ for some $\gamma>0$. Then as $n \rightarrow \infty$

$$
E O(G)=\Theta_{k_{1}, k_{2}}\left(2^{|E G|+\frac{n}{2}} \pi^{-\frac{n}{2}} / \sqrt{t(G)}\right),
$$

where $t(G)$ enotes the number of spanning trees of the graph $G$ and constants $k_{1}, k_{2}>0$ depend only on $\sigma$.

Remark 2.1. Taking into account (1.4), the value $t(G)$ can be represented as the principal minor of the Laplacian matrix $Q$.

Remark 2.2. For the complete graph $\lambda_{1}=n, E K_{n}=\frac{n(n-1)}{2}$ and $t\left(K_{n}\right)=$ $n^{n-2}$. The result of Theorem 2.1 for this case is in agreement with asymptotic formula (1.1).

Remark 2.3. There is the result on the asymptotic behavior of Eulerian circuits analogous to Theorem 2.1, see [1].

\section{Some basic properties of the Laplacian matrix}

In what follows we suppose that

$$
G \text { is a simple graph. }
$$

The Laplacian matrix $Q$ of the graph $G$ has the eigenvector $[1,1, \ldots, 1]^{T}$, corresponding to the eigenvalue $\lambda_{0}=0$. We use notation $\hat{Q}=Q+J$, where $J$ denotes the matrix with every entry 1 . Note that $Q$ and $\hat{Q}$ have the same set of eigenvectors and eigenvalues, except for the eigenvalue corresponding to the eigenvector $[1,1, \ldots, 1]^{T}$, which equals 0 for $Q$ and $n$ for $\hat{Q}$.

Using (2.4), we get that

$$
\lambda_{n}=\|Q\|_{2} \leq\|\hat{Q}\|_{2} \leq\|\hat{Q}\|_{1}=\max _{j} \sum_{k=1}^{n}\left|\hat{Q}_{j k}\right|=n .
$$

We denote by $G_{r}$ the graph which arises from $G$ by removing vertices $v_{1}, v_{2}, \ldots, v_{r}$ and all adjacent edges.

Lemma 3.1. Let condition (3.1) holds for graph $G$ with $n$ vertices. Then

$$
\lambda_{2}(G) \leq \frac{n}{n-1} \min _{j} d_{j}
$$




$$
\lambda_{2}\left(G_{r}\right) \geq \lambda_{2}(G)-r
$$

where $\lambda_{1}(G)$ is the algebraic connectivity of $G$ and $d_{j}$ is the degree of the vertex $v_{j} \in V G$.

The proof of Lemma 3.1 can be found in 2].

Lemma 3.2. Let $a>0$ and $I$ be identity $n \times n$ matrix. Then for any $n$ and $n \times n$ symmetric matrix $X$ such that the matrix $I+X$ is nonsingular and $\left|X_{i j}\right| \leq a / n$,

$$
\mu_{2}(I+X) \leq \mu_{\infty}(I+X) \leq C \mu_{2}(I+X),
$$

where $C$ depends only on a. (does not depend on $n$ )

Proof of Lemma 3.2. The left-hand side of (3.5) follows from (2.4). We order the eigenvalues of $I+X$ modulo

$$
\left|\chi_{1}\right| \leq\left|\chi_{2}\right| \leq \ldots \leq\left|\chi_{n}\right| .
$$

Using (2.4), we get that

$$
\left|\chi_{n}\right|=\|I+X\|_{2} \leq\|I+X\|_{\infty} \leq\|I\|_{\infty}+\|X\|_{\infty} \leq 1+a .
$$

We consider $\vec{x}=\left(x_{1}, \ldots, x_{n}\right) \in \mathbb{R}^{n}$ such that $\|\vec{x}\|_{\infty}=1$. For simplicity, we assume that $x_{1}=\|\vec{x}\|_{\infty}=1$. We denote by $\mathcal{J}=\left\{j \mid x_{j}>\frac{1}{2 a}\right\}$. that

Case 1. $|\mathcal{J}|<\frac{n}{4 a}$. Estimating the first coordinate of $(I+X) \vec{x}$, we get

$$
\begin{array}{r}
\|(I+X) \vec{x}\|_{\infty} \geq x_{1}-\frac{a}{n}\left(\sum_{j \in \mathcal{J}}\left|x_{j}\right|+\sum_{j \notin \mathcal{J}}\left|x_{j}\right|\right) \geq \\
\geq 1-\frac{a}{n}\left(\frac{n}{4 a} \cdot 1+n \cdot \frac{1}{2 a}\right)=\frac{1}{4}\|\vec{x}\|_{\infty} .
\end{array}
$$

Case 2. $|\mathcal{J}| \geq \frac{n}{4 a}$. Note that

$$
\sqrt{n\|(I+X) \vec{x}\|_{\infty}^{2}} \geq\|(I+X) \vec{x}\|_{2} \geq\left|\chi_{1}\right| \cdot\|\vec{x}\|_{2} \geq\left|\chi_{1}\right| \cdot \sqrt{|\mathcal{J}| \cdot \frac{1}{4 a^{2}}}\|\vec{x}\|_{\infty} .
$$

Then

$$
\|(I+X) \vec{x}\|_{\infty} \geq \frac{\left|\chi_{1}\right|}{4 a^{3 / 2}}\|\vec{x}\|_{\infty} .
$$

Combining (3.8) (3.10), we get that at least one of the following inequalities holds.

$$
\left\|(I+X)^{-1}\right\|_{\infty} \leq 4 \quad\left\|(I+X)^{-1}\right\|_{\infty} \leq \frac{4 a^{3 / 2}}{\left|\chi_{1}\right|} .
$$

From (3.7) we have that

$$
\|I+X\|_{\infty} \leq 1+a .
$$

Taking into account $\left|\chi_{n}\right| \leq 1+a$ and $\mu_{2}(I+X)=\frac{\left|\chi_{n}\right|}{\left|\chi_{1}\right|} \geq 1$, we obtain (3.5)

The proofs of Lemma 4.1, Lemma 4.2 and Lemma 4.3 are based on the following property of the Laplacian matrix. 
Corollary 3.1. Let $G$ be a simple graph with $n$ vertices and algebraic connectivity of the graph $\lambda_{2} \geq \gamma n$ for some $\gamma>0$. Then there is some constant $c_{\infty}>0$, depending only on $\gamma$, such that

$$
\left\|\hat{Q}^{-1}\right\|_{1}=\left\|\hat{Q}^{-1}\right\|_{\infty} \leq \frac{c_{\infty}}{n} .
$$

Proof of Corollary 3.1. Using (3.3), we get that

$$
d_{j} \geq \lambda_{2} \frac{n-1}{n} \geq \gamma(n-1) \geq \gamma n / 2 .
$$

Taking into account (3.2), all eigenvalues of $\hat{Q}$ are in the interval $[\gamma n ; n]$. Inequality (3.13) follows easily from the assertion of Lemma 3.2 for the matrix $\Omega^{T} \hat{Q} \Omega$, where

$$
\Omega_{j k}=\left\{\begin{array}{cl}
\frac{1}{\sqrt{d_{j}+1}}, & \text { if } j=k \\
0, & \text { otherwise }
\end{array}\right.
$$

The following lemma will be applied to estimate the determinant of a matrix close to the identity matrix $I$.

Lemma 3.3. Let $X$ be an $n \times n$ matrix such that $\|X\|_{2}<1$. Then for fixed $m \geq 2$

$$
\operatorname{det}(I+X)=\exp \left(\sum_{r=1}^{m-1} \frac{(-1)^{r+1}}{r} \operatorname{tr}\left(X^{r}\right)+E_{m}(X)\right),
$$

where tr is the trace function and

$$
\left|E_{m}(X)\right| \leq \frac{n}{m} \frac{\|X\|_{2}^{m}}{1-\|X\|_{2}}
$$

The proof of Lemma 3.3 is based on evaluating the trace of the matrix $\ln (I+X)$, using the representation as a convergent series. Lemma 3.3 was also formulated and proved in 8$]$.

Lemma 3.4. Let $G$ be a simple graph with $n$ vertices and algebraic connectivity of the graph $\lambda_{2} \geq \gamma n$ for some $\gamma>0$. et $G_{1}$ be the graph which arises from $G$ by removing vertex $v_{1}$ and all adjacent edges. Then there is a constant $c>0$ depending only on $\gamma$ such that

$$
\operatorname{det} \hat{Q}_{1} \geq \frac{\operatorname{det} \hat{Q}}{c n} .
$$

Proof of Lemma 3.4. Note that the matrix $M_{11}$ that results from deleting the first row and the first column of $\hat{Q}$ coincides with the matrix $\hat{Q}_{1}$ with the exception of the diagonal elements. Let $\Omega$ be a diagonal matrix such that

$$
\Omega_{j j}= \begin{cases}1, & \text { if }\left\{v_{1}, v_{j}\right\} \in E G, \\ 0, & \text { otherwise. }\end{cases}
$$

Define $n \times n$ matrix $X$ by

$$
X_{j k}=\left\{\begin{array}{cl}
\frac{1}{d_{1}+1}, & \left\{v_{1}, v_{j}\right\} \notin E G,\left\{v_{1}, v_{k}\right\} \notin E G, \text { and } j, k \neq 1 \\
0, & \text { otherwise. }
\end{array}\right.
$$


After performing one step of the Gaussian elimination for $\hat{Q}+\Omega+X$, we obtain that

$$
\operatorname{det}(\hat{Q}+\Omega+X)=\left(d_{1}+1\right) \operatorname{det} \hat{Q}_{1},
$$

Using (2.4), (3.14), we have that

$$
\|\Omega+X\|_{2} \leq\|\Omega\|_{2}+\|X\|_{2} \leq\|\Omega\|_{2}+\|X\|_{1} \leq 1+\frac{n}{d_{1}+1} \leq \frac{3}{\gamma} .
$$

Since the algebraic connectivity $\lambda_{2} \geq \gamma n$

$$
\left\|(\Omega+X) \hat{Q}^{-1}\right\|_{2} \leq\|\Omega+X\|_{2}\left\|\hat{Q}^{-1}\right\|_{2} \leq \frac{3}{\gamma \lambda_{2}} \leq \frac{3}{\gamma^{2} n}
$$

Combining Lemma 3.3 and (3.23), we get that as $n \rightarrow \infty$

$$
\begin{array}{r}
\operatorname{det}\left(I+(\Omega+X) \hat{Q}^{-1}\right)=\exp \left(\operatorname{tr}\left((\Omega+X) \hat{Q}^{-1}\right)+E_{2}\left((\Omega+X) \hat{Q}^{-1}\right)\right) \geq \\
\geq \exp \left(-n \frac{3}{\gamma^{2} n}+O\left(n^{-1}\right)\right) .
\end{array}
$$

Using (3.21) and (3.24) we get that as $n \rightarrow \infty$

$$
\begin{aligned}
\left(d_{1}+1\right) \operatorname{det} \hat{Q}_{1} & =\operatorname{det}\left(I+(\Omega+X) \hat{Q}^{-1}\right) \operatorname{det} \hat{Q} \geq \\
& \geq \operatorname{det} \hat{Q} \exp \left(-3 / \gamma^{2}+O\left(n^{-1}\right)\right) .
\end{aligned}
$$

Since $d_{1}+1 \leq n$, we obtain (3.18).

Corollary 3.2. Let the assumptions of Lemma 3.4 hold. Let $G_{r}$ be the graph which arises from $G$ by removing vertices $v_{1}, v_{2}, \ldots, v_{r}$ and all adjacent edges. Then there is a constant $c_{1}>0$ depending only on $\gamma$ such that

$$
\operatorname{det} \hat{Q}\left(G_{r}\right) \geq \frac{\operatorname{det} \hat{Q}(G)}{\left(c_{1} n\right)^{r}}
$$

for any $r \leq \gamma n / 2$.

Proof of Corollary 3.2. From (3.4) we have that

$$
\lambda_{2}\left(G_{r}\right) \geq \gamma n-r \geq \gamma n / 2 .
$$

Using $r$ times the assertion of the Lemma 3.4, we obtain (3.26).

According to (1.4)

$$
t(G)=\frac{1}{n} \lambda_{2} \lambda_{2} \cdots \lambda_{n-1}=\frac{\operatorname{det} \hat{Q}}{n^{2}},
$$

then the following proposition holds.

Corollary 3.3. Let the assumptions of Lemma 3.4 hold. Then there is a constant $c>0$ depending only on $\gamma$ such that

$$
t\left(G_{1}\right) \geq \frac{t(G)}{c n}
$$

where $t(G)$ denotes the number of spanning trees of the graph $G$. 
Lemma 3.5. Let $a>0$ and the assumptions of Lemma 3.4 hold. Then for any set $A \subset V G$ such, that $|A| \geq$ an, there is a function $h: V G \rightarrow \mathbb{N}_{0}$, having following properties:

$$
\begin{gathered}
h(v)=0, \quad v \in A, \quad h(v) \leq H, \quad v \in V G \\
|\{w \in V G \mid(w, v) \in E G \quad h(w)<h(v)\}| \geq \alpha n, \quad v \notin A,
\end{gathered}
$$

where constants $H, \alpha>0$ depend only on a and $\gamma$.

Proof of 3.5. At first, we construct the set $A_{1}=\{v \in V G \mid h(v)=1\}$, having property (3.31).

If $|A|>n-\gamma n / 4$, then let $A_{1}=\{v \in V G \mid v \notin A\}$. Taking into account (3.14), we get that property (3.31) hold for $\alpha=\gamma / 4$. In this case $H=1$.

For $|A| \leq n-\gamma n / 4$ define $\vec{x} \in \mathbb{R}^{n}$ such that

$$
x_{j}=\left\{\begin{array}{cc}
1-|A| / n, & v_{j} \in A, \\
-|A| / n, & v_{j} \notin A .
\end{array}\right.
$$

Since $x_{1}+x_{2}+\ldots+x_{n}=0$

$$
\begin{aligned}
\vec{x}^{T} Q \vec{x}=\vec{x}^{T} \hat{Q} \vec{x} \geq \lambda_{2}\|\vec{x}\|_{2}^{2} & \geq \lambda_{2}|A|\left(\frac{n-|A|}{n}\right)^{2} \geq \\
& \geq \gamma n \operatorname{an}(\gamma / 4)^{2}=\frac{a \gamma^{3} n^{2}}{16} .
\end{aligned}
$$

On the other hand,

$$
\vec{x}^{T} Q \vec{x}=\sum_{\left\{v_{j}, v_{k}\right\} \in E G}\left(x_{j}-x_{k}\right)^{2},
$$

which is equal to the number of edges $(v, w) \in E G$, where $v \in A, w \notin A$. We denote $A_{1}$ the set of vertices $w \notin A$, having at least $\alpha n$ adjacent vertices in $A$, where $\alpha=\frac{1}{32} a \gamma^{3}$.

$$
\vec{x}^{T} Q \vec{x} \leq n\left|A_{1}\right|+\alpha n|V G| .
$$

Combining (3.33) and (3.35), we get that $\left|A_{1}\right| \geq \alpha n$.

We make further construction of the function $h$ inductively, using for the $k$-th step the set $A^{(k)}=A \cup A_{1} \cup \ldots \cup A_{k}$. The number of steps does not exceed $1 / \alpha$ as $\left|A_{k}\right| \geq \alpha n$ for each step, perhaps with the exception of the last one.

\section{Proof of Theorem 2.1}

In a similar way as in 9] (see the proof of Theorem 3.1) we note that the function

$$
\prod_{\left\{v_{j}, v_{k}\right\} \in E G}\left(x_{j}{ }^{-1} x_{k}+x_{k}{ }^{-1} x_{j}\right)
$$

is the generating function the number of orientations of graph $G$ by the differences in the numbers of incoming and outgoing edges at each vertex. The value 
$E O(G)$ is the constant term, which we can extract via Cauchys Theorem using the unit circle as a contour for each variable:

$$
E O(G)=\frac{1}{(2 \pi i)^{n}} \oint \cdots \oint \frac{\prod_{\left\{v_{j}, v_{k}\right\} \in E G}\left(x_{j}^{-1} x_{k}+x_{k}{ }^{-1} x_{j}\right)}{x_{1} x_{2} \cdots x_{n}} d x_{1} d x_{2} \ldots d x_{n} .
$$

Making the substitution $x_{j}=e^{i \theta_{j}}$ for each $j$, we find that

$$
E O(G)=2^{|E G|} \pi^{-n} S, \quad S=\int_{U_{n}(\pi / 2)} \prod_{\left\{v_{j}, v_{k}\right\} \in E G} \cos \Delta_{j k} d \vec{\theta},
$$

where $\Delta_{j k}=\theta_{j}-\theta_{k}$,

$$
U_{n}(\rho)=\left\{\left(x_{1}, x_{2}, \ldots, x_{n}\right)|| x_{j} \mid \leq \rho \quad j\right\}
$$

and using the fact that for graphs with vertices of even degree the integrand is unchanged by the substitutions $\theta_{j} \rightarrow \theta_{j}+\pi$.

Let's start the evaluation $S$ from the part that makes a major contribution to the integral We fix some sufficiently small constant $\varepsilon>0$. Let

$$
V_{0}=\left\{\vec{\theta} \in U_{n}(\pi / 2)|| \theta_{j}-\bar{\theta} \mid(\bmod \pi) \leq n^{-1 / 2+\varepsilon}, \quad \bar{\theta}=\frac{\theta_{1}+\ldots+\theta_{n}}{n}\right\} .
$$

By Taylors theorem we have that for $\vec{\theta} \in V_{0}$

$$
\prod_{\left\{v_{j}, v_{k}\right\} \in E G} \cos \Delta_{j k}=\exp \left(-\frac{1}{2} \sum_{\left\{v_{j}, v_{k}\right\} \in E G} \Delta_{j k}^{2}-\frac{1}{12} \sum_{\left\{v_{j}, v_{k}\right\} \in E G} \Delta_{j k}^{4}+O\left(n^{-1+6 \varepsilon}\right)\right) .
$$

We denote by $S_{0}$ the contribution to $S$ in the integration over the region $V_{0}$.

Lemma 4.1. Let $G$ be a simple graph with $n$ vertices and the algebraic connectivity $\lambda_{2} \geq \gamma n$ for some $\gamma>0$. Then for any $a, b>0$ as $n \rightarrow \infty$

$$
\int_{V_{0}} \exp \left(-a \sum_{\left\{v_{j}, v_{k}\right\} \in E G} \Delta_{j k}^{2}-b \sum_{\left\{v_{j}, v_{k}\right\} \in E G} \Delta_{j k}^{4}\right) d \vec{\theta}=\Theta_{k_{1}, k_{2}}\left(n \int_{\mathbb{R}^{n}} e^{-a \vec{\theta}^{T} \hat{Q} \vec{\theta}} d \vec{\theta}\right),
$$

where constants $k_{1}, k_{2}>0$ depend only on $a, b$ and $\gamma$.

Lemma 4.1 follows from Lemma 8.3 of [1]. The proof is given in Section 5 .

Combining (3.28), (4.3), (4.6), (4.7) and

$$
\int_{\mathbb{R}^{n}} e^{-a \vec{\theta}^{T} \hat{Q} \vec{\theta}} d \vec{\theta}=\pi^{n / 2} a^{-n / 2} / \sqrt{\operatorname{det} \hat{Q}}
$$

we get that

$$
S_{0}=\Theta_{k_{1}, k_{2}}\left(2^{\frac{n-1}{2}} \pi^{\frac{n+1}{2}} / \sqrt{t(G)}\right)
$$

where constants $k_{1}, k_{2}>0$ depend only on $\gamma$. 
Thus it remains to show that the other parts are negligible One can show that

$$
|\cos (x)| \leq \exp \left(-\frac{1}{2} x^{2}\right) \text { for }|x| \leq \frac{9}{16} \pi .
$$

Divide the interval $\left[-\frac{1}{2} \pi, \frac{1}{2} \pi\right]$ mod $\pi$ into 32 equal intervals $H_{0}, \ldots, H_{31}$ such that $H_{0}=\left[-\frac{1}{64} \pi, \frac{1}{64} \pi\right]$. For each $j$, define the region $W_{j} \subseteq U_{n}(\pi / 2)$ as the set of points $\vec{\theta} \in U_{n}(\pi / 2)$, having at least $\frac{1}{32} n$ coordinates in $H_{j}$. Clearly, the $W_{j} \mathrm{~s}$ cover $U_{n}(\pi / 2)$ and also each $W_{j}$ can be mapped to $W_{0}$ by a uniform translation of the $\theta_{j} \bmod \pi$. This mapping preserves the integrand of (4.3), and also maps $V_{0}$ to itself, so we have that

$$
\int_{U_{n}(\pi / 2)-V_{0}} \prod_{\left\{v_{j}, v_{k}\right\} \in E G} \cos \Delta_{j k} d \vec{\theta} \leq 32 Z,
$$

where

$$
Z=\int_{W_{0}-V_{0}} \prod_{\left\{v_{j}, v_{k}\right\} \in E G}\left|\cos \Delta_{j k}\right| d \vec{\theta} .
$$

We proceed by defining integrals $S_{1}, S_{2}, S_{3}$ in such a way that $Z$ is obviously bounded by their sum. We then show that each of them is negligible. Let

$$
\begin{aligned}
& V_{1}=\left\{\vec{\theta} \in W_{0}|| \theta_{j} \mid \geq \frac{1}{32} \pi \text { for fewer than } n^{\varepsilon} \text { values of } j\right\}, \\
& V_{2}=\left\{\vec{\theta} \in V_{1}|| \theta_{j} \mid \geq \frac{1}{16} \pi \text { for at least one value of } j\right\} .
\end{aligned}
$$

Then our three integrals can be defined as

$$
\begin{aligned}
S_{1} & =\int_{W_{0}-V_{1}} \prod_{\left\{v_{j}, v_{k}\right\} \in E G}\left|\cos \Delta_{j k}\right| d \vec{\theta}, \\
S_{2} & =\int_{V_{2}} \prod_{\left\{v_{j}, v_{k}\right\} \in E G}\left|\cos \Delta_{j k}\right| d \vec{\theta}, \\
S_{3} & =\int_{V_{1}-V_{2}-V_{0}} \prod_{\left\{v_{j}, v_{k}\right\} \in E G}\left|\cos \Delta_{j k}\right| d \vec{\theta} .
\end{aligned}
$$

We begin with $S_{1}$. Let $h$ be the function from Lemma 3.5 for the set $A=$ $\left\{v_{j}|| \theta_{j} \mid \leq \frac{1}{64} \pi\right\}$. We denote $l_{\text {min }}$ such natural number that inequality

$$
\left|\theta_{j}\right| \geq \frac{1}{64} \pi(1+l / H)
$$

holds for at least $n^{\varepsilon} / H$ indices of the set $\left\{j \mid h\left(v_{j}\right)=l\right\}$. Existence of $l_{\min }$ follows from the definition of the region $V_{1}$. If $\theta_{j}$ and $\theta_{k}$ are such that

$$
\left|\theta_{j}\right| \geq \frac{1}{64} \pi\left(1+l_{\text {min }} / H\right) \text { and }\left|\theta_{k}\right| \leq \frac{1}{64} \pi\left(1+\left(l_{\text {min }}-1\right) / H\right)
$$

or vice versa, we have that $\left|\cos \Delta_{j k}\right| \leq \cos \left(\frac{1}{64} \pi / H\right)$. This includes at least $\left(\alpha n-n^{\varepsilon}\right) n^{\varepsilon} / H$ edges $\left\{v_{j}, v_{k}\right\} \in E G$. Using (3.2) and (3.28), we get that as $n \rightarrow \infty$

$$
S_{1} \leq \pi^{n}\left(\cos \frac{\pi}{64 H}\right)^{\left(\alpha n-n^{\varepsilon}\right) n^{\varepsilon} / H}=O\left(\exp \left(-c n^{1+\varepsilon}\right)\right) 2^{\frac{n-1}{2}} \pi^{\frac{n+1}{2}} / \sqrt{t(G)}
$$


for some constant $c>0$, depending only on $\gamma$.

For $1 \leq r \leq n^{\varepsilon}$ let $S_{2}(r)$ denote the contribution to $S_{2}$ of those $\theta \in V_{2}$ such that $\left|\theta_{j}\right| \geq \frac{1}{16} \pi$ for exactly $r$ values of $j$. If $\left|\theta_{j}\right| \leq \frac{1}{32} \pi$ and $\left|\theta_{k}\right| \geq \frac{1}{16} \pi$ or vice versa, we have that

$$
\left|\cos \Delta_{j k}\right| \leq \cos \left(\frac{1}{32} \pi\right)
$$

This includes at least $r\left(\gamma n / 2-n^{\varepsilon}\right)$ edges $\left\{v_{j}, v_{k}\right\} \in E G$, because the degree of any vertex of the graph $G$ is at least $\gamma n / 2$, see (3.14). For pairs $(j, k)$ such that $\left|\theta_{j}\right|,\left|\theta_{k}\right| \leq \frac{1}{16} \pi$, we use (4.10). We put $\overrightarrow{\theta^{\prime}}=\left(\theta_{1}, \ldots, \theta_{n-r}\right)$.

$$
S_{2}(r) \leq \pi^{r}\left(\cos \frac{\pi}{32}\right)^{r\left(\gamma n / 2-n^{\varepsilon}\right)} \sum_{G_{r}} \int_{U_{n-r}(\pi / 2)} \exp \left(-\frac{1}{2} \sum_{\left\{v_{j}, v_{k}\right\} \in E G_{r}} \Delta_{j k}^{2}\right) d \overrightarrow{\theta^{\prime}},
$$

where the first sum is over graphs, arises from $G$ by removing all possible sets of $r$ vertices.

Lemma 4.2. Let the assumptions of Lemma 4.1 hold. Then

$$
\int_{U_{n}(\pi / 2)} \exp \left(-\frac{1}{2} \sum_{\left\{v_{j}, v_{k}\right\} \in E G} \Delta_{j k}^{2}\right) d \vec{\theta} \leq \frac{2^{\frac{n-1}{2} \pi^{\frac{n+1}{2}} n}}{\sqrt{\operatorname{det} \hat{Q}}} .
$$

Lemma 4.2 was formulated and proved in [1] (see Lemma 6.1). The proof is given also in Section 5.

Using Lemma 3.1 and Corollary 3.2, we get that

$$
\begin{gathered}
\lambda_{2}\left(G_{r}\right) \geq \gamma n-n^{\varepsilon}, \\
\operatorname{det} \hat{Q}\left(G_{r}\right) \geq \frac{\operatorname{det} \hat{Q}}{\left(c_{1} n\right)^{r}} .
\end{gathered}
$$

According to Lemma 4.2 we have that

$$
\int_{U_{n-r}(\pi / 2)} \exp \left(-\frac{1}{2} \sum_{\left\{v_{j}, v_{k}\right\} \in E G_{r}} \Delta_{j k}^{2}\right) d \overrightarrow{\theta^{\prime}} \leq \frac{2^{\frac{n-r-1}{2}} \pi^{\frac{n-r+1}{2}} n}{\sqrt{\operatorname{det} \hat{Q}\left(G_{r}\right)}} .
$$

Combining (4.19) with (4.22), (4.23) and allowing $n^{r}$ for the choice of the set of $r$ vertices for $G_{r}$, we get that

$$
S_{2}(r) \leq 2^{\frac{n-r-1}{2}} \pi^{\frac{n+r+1}{2}} n^{r+1}\left(\cos \frac{\pi}{32}\right)^{r\left(\gamma n / 2-n^{\varepsilon}\right)} \frac{\left(c_{1} n\right)^{r / 2}}{\sqrt{\operatorname{det} \hat{Q}}} .
$$

Using (3.2) and summing over $0 \leq r \leq n^{\varepsilon}$, we find that

$$
S_{2}=\sum_{r=1}^{n^{\varepsilon}} S_{2}(r)=O\left(c^{-n}\right) 2^{\frac{n-1}{2}} \pi^{\frac{n+1}{2}} / \sqrt{t(G)}
$$

for some constant $c>1$, depending only on $\gamma$. 
Note that $\Delta_{j k} \leq \frac{1}{8} \pi$ for $\vec{\theta} \in V_{1}-V_{2}$, thus

$$
V_{1}-V_{2} \subset V_{3}=\left\{\vec{\theta} \in U_{n}(\pi / 2)|| \theta_{j}-\bar{\theta} \mid(\bmod \pi) \leq \pi / 8\right\}
$$

where

$$
\bar{\theta}=\frac{\theta_{1}+\ldots+\theta_{n}}{n} .
$$

Since the integrand is invariant under uniform translation of all the $\theta_{j}$ 's $\bmod \pi$ as well as $V_{0}$ and $V_{3}$ are mapped into itself, we can fix $\bar{\theta}=0$ and multiply it by the ratio of its range $\pi$ to the length $n^{-1 / 2}$ of the vector $\frac{1}{n}[1,1, \ldots, 1]^{T}$. Thus we get that

$$
S_{3} \leq \pi n^{1 / 2} \int_{L \cap U_{n}(\pi / 8) \backslash V_{0}} \prod_{\left\{v_{j}, v_{k}\right\} \in E G}\left|\cos \Delta_{j k}\right| d L,
$$

where $L$ denotes the orthogonal complement to the vector $[1,1, \ldots, 1]^{T}$. In a similar way as in (4.19) we find that

$$
S_{3} \leq \pi n^{1 / 2} \int_{L \cap U_{n}(\pi / 8) \backslash V_{0}} \exp \left(-\frac{1}{2} \sum_{\left\{v_{j}, v_{k}\right\} \in E G} \Delta_{j k}^{2}\right) d L .
$$

Lemma 4.3. Let the assumptions of Lemma 4.1 hold. Then as $n \rightarrow \infty$

$$
\int_{L \backslash U_{n}\left(n^{-1 / 2+\varepsilon}\right)} \exp \left(-\frac{1}{2} \sum_{\left\{v_{j}, v_{k}\right\} \in E G} \Delta_{j k}^{2}\right) d L=O\left(\exp \left(-c n^{2 \varepsilon}\right)\right) \frac{2^{\frac{n-1}{2}} \pi^{\frac{n-1}{2}} n^{1 / 2}}{\sqrt{\operatorname{det} \hat{Q}}}
$$

for some $c>0$, depending only on $\gamma$.

Lemma 4.3 was formulated and proved in [1] (see Lemma 6.2). The proof is given also in Section 5 .

Combining (3.28), (4.30) and (4.29), we get that as $n \rightarrow \infty$

$$
S_{3}=O\left(\exp \left(-c n^{2 \varepsilon}\right)\right) \frac{2^{\frac{n-1}{2}} \pi^{\frac{n+1}{2}} n}{\sqrt{\operatorname{det} \hat{Q}}}=O\left(\exp \left(-c n^{2 \varepsilon}\right)\right) 2^{\frac{n-1}{2}} \pi^{\frac{n+1}{2}} / \sqrt{t(G)}
$$

for some $c>0$, depending only on $\gamma$. Combining (4.9), (4.17), (4.25) and (4.31), we obtain (2.8) as well as the following lemma.

Lemma 4.4. Let the assumptions of Lemma 4.1 hold. Then as $n \rightarrow \infty$

$$
S=\left(1+O\left(\exp \left(-c n^{2 \varepsilon}\right)\right)\right) S_{0}
$$

for some $c>0$, depending only on $\gamma$.

\section{Proofs of Lemmas $4.1-4.3$}

In this section we always assume that the assumptions of Lemma 4.1 hold. Let

$$
\vec{\phi}=\vec{\phi}(\vec{\theta})=\left[\phi_{1}(\vec{\theta}), \ldots, \phi_{n}(\vec{\theta})\right]^{T}=\hat{Q} \vec{\theta} .
$$


Let $P(\vec{\theta})$ be the orthogonal projection $\vec{\theta}$ onto the space $L$, where $L$ denotes the orthogonal complement to the vector $[1,1, \ldots, 1]^{T}$. Note that

$$
Q \vec{\theta}=Q P(\vec{\theta})
$$

For any $a>0$, we have that:

$$
\int_{\mathbb{R}^{n}} e^{-a \vec{\theta}^{T} \hat{Q} \vec{\theta}} d \vec{\theta}=\pi^{n / 2} a^{-n / 2} / \sqrt{\operatorname{det} \hat{Q}}
$$

and

$$
\int_{L} e^{-a \vec{\theta}^{T} \hat{Q} \vec{\theta}} d L=\int_{L} e^{-a \vec{\theta}^{T} Q \vec{\theta}} d L=\pi^{\frac{n-1}{2}} a^{-\frac{n-1}{2}} n^{1 / 2} / \sqrt{\operatorname{det} \hat{Q}} .
$$

Proof of Lemma 4.2. Note that

$$
\sum_{\left\{v_{j}, v_{k}\right\} \in E G} \Delta_{j k}^{2}=\vec{\theta}^{T} Q \vec{\theta} .
$$

The diagonal of the cube $U_{n}(\pi / 2)$ is equal to $\pi n^{1 / 2}$. Using (5.2), we find that

$$
\int_{U_{n}(\pi / 2)} \exp \left(-\frac{1}{2} \sum_{\left\{v_{j}, v_{k}\right\} \in E G} \Delta_{j k}^{2}\right) d \vec{\theta} \leq \pi n^{1 / 2} \int_{L} e^{-\frac{1}{2} \vec{\theta}^{T} Q \vec{\theta}} d L .
$$

Combining (5.4) and (5.6), we obtain (4.20).

Note that for some function $g_{1}(\vec{\theta})=g_{1}\left(\theta_{2}, \ldots, \theta_{n}\right)$

$$
\vec{\theta}^{T} \hat{Q} \vec{\theta}=\frac{\phi_{1}(\vec{\theta})^{2}}{d_{1}+1}+g_{1}(\vec{\theta}) .
$$

We recall that (see (3.14) $)$

$$
\min _{j} d_{j} \geq \gamma n / 2 .
$$

Combining (5.7) and (5.8), we get that as $n \rightarrow \infty$

$$
\begin{aligned}
\int_{\mathbb{R}^{n}} e^{-a \vec{\theta}^{T} \hat{Q} \vec{\theta}} d \vec{\theta} & =\int_{-\infty}^{+\infty} \cdots \int_{-\infty}^{+\infty} e^{-a g_{1}\left(\theta_{2}, \ldots, \theta_{n}\right)}\left(\int_{-\infty}^{+\infty} e^{-a \frac{\phi_{1}(\vec{\theta})^{2}}{d_{1}+1}} d \theta_{1}\right) d \theta_{2} \ldots d \theta_{n} \\
& =\left(1+O\left(\exp \left(-\tilde{c} n^{2 \varepsilon}\right)\right)\right) \int_{\left|\phi_{1}(\vec{\theta})\right| \leq \frac{1}{2} c_{\infty}^{-1} n^{1 / 2+\varepsilon}} e^{-a \vec{\theta}^{T} \hat{Q} \vec{\theta}} d \vec{\theta}
\end{aligned}
$$

for some $\tilde{c}>0$, depending obly on $\gamma$ and $a$, where $c_{\infty}$ is the constant of (3.13). Combining similar to (5.9) expressions for $\phi_{1}, \ldots \phi_{n}$, we find that as $n \rightarrow \infty$

$$
\int_{\|\vec{\phi}(\vec{\theta})\|_{\infty} \leq \frac{1}{2} c_{\infty}^{-1} n^{1 / 2+\varepsilon}} e^{-a \vec{\theta}^{T} \hat{Q} \vec{\theta}} d \vec{\theta}=\left(1+O\left(\exp \left(-c n^{2 \varepsilon}\right)\right)\right) \int_{\mathbb{R}^{n}} e^{-a \vec{\theta}^{T} \hat{Q} \vec{\theta}} d \vec{\theta}
$$


for some $c>0$, depending only on $\gamma a$. Using (5.10) and (3.13), we get that as $n \rightarrow \infty$

$$
\int_{U_{n}\left(\frac{1}{2} n^{-1 / 2+\varepsilon}\right)} e^{-a \vec{\theta}^{T} \hat{Q} \vec{\theta}} d \vec{\theta}=\left(1+O\left(\exp \left(-c n^{2 \varepsilon}\right)\right)\right) \int_{\mathbb{R}^{n}} e^{-a \vec{\theta}^{T} \hat{Q} \vec{\theta}} d \vec{\theta} .
$$

Proof of Lemma 4.3. Note that

$$
\|P(\vec{\theta})\|_{\infty}=\left\|\vec{\theta}-\bar{\theta}[1,1, \ldots, 1]^{T}\right\|_{\infty} \leq 2\|\vec{\theta}\|_{\infty},
$$

where

$$
\bar{\theta}=\frac{\theta_{1}+\theta_{2}+\ldots \theta_{n}}{n} .
$$

Thus

$$
U_{n}\left(\frac{1}{2} n^{-1 / 2+\varepsilon}\right) \subset\left\{\vec{\theta} \mid P(\vec{\theta}) \in U_{n}\left(n^{-1 / 2+\varepsilon}\right)\right\}
$$

Using (5.2), (5.5) and (5.14), we find that

$$
\begin{aligned}
& \int_{L \cap U_{n}\left(n^{-1 / 2+\varepsilon}\right)} \exp \left(-\frac{1}{2} \sum_{\left\{v_{j}, v_{k}\right\} \in E G} \Delta_{j k}^{2}\right) d L=\int_{L \cap U_{n}\left(n^{-1 / 2+\varepsilon}\right)} e^{-\frac{1}{2} \vec{\theta}^{T} Q \vec{\theta}} d L= \\
= & \int_{P(\vec{\theta}) \in U_{n}\left(n^{-1 / 2+\varepsilon}\right)} e^{-\frac{1}{2} \vec{\theta}^{T} \hat{Q} \vec{\theta}} d \vec{\theta} / \int_{-\infty}^{+\infty} e^{-\frac{1}{2} n x^{2}} d x \geq \frac{n^{1 / 2}}{\sqrt{2 \pi}} \int_{U_{n}\left(\frac{1}{2} n^{-1 / 2+\varepsilon}\right)} e^{-\frac{1}{2} \vec{\theta}^{T} \hat{Q} \vec{\theta}} d \vec{\theta} .
\end{aligned}
$$

Combining (5.3), (5.11) and (5.15), we obtain (4.30).

Proposition 5.1. As $n \rightarrow \infty$

$$
\begin{aligned}
\int_{U_{n}\left(n^{-1 / 2+\varepsilon}\right)} \exp \left(-a \vec{\theta}^{T} \hat{Q} \vec{\theta}-b \sum_{\left\{v_{j}, v_{k}\right\} \in E G} \Delta_{j k}^{4}\right) & d \vec{\theta}= \\
& =\Theta_{k_{1}, k_{2}}\left(\int_{\mathbb{R}^{n}} e^{-a \vec{\theta}^{T} \hat{Q} \vec{\theta}} d \vec{\theta}\right),
\end{aligned}
$$

where constants $k_{1}, k_{2}>0$ depend only on $a, b$ and $\gamma$.

In th present paper we give only the scheme of the proof of Proposition 5.1. The detailed proof can be found in [1] (see Lemma 8.3).

Scheme of the proof of Proposition 5.1. Since $a, b>0$

$$
\int_{U_{n}\left(n^{-1 / 2+\varepsilon}\right)} \exp \left(-a \vec{\theta}^{T} \hat{Q} \vec{\theta}-b \sum_{\left\{v_{j}, v_{k}\right\} \in E G} \Delta_{j k}^{4}\right) d \vec{\theta} \leq \int_{\mathbb{R}^{n}} e^{-a \vec{\theta}^{T} \hat{Q} \vec{\theta}} d \vec{\theta} .
$$

Let

$$
R_{k}(\vec{\theta})=8 b n \sum_{j=k}^{n} \theta_{j}^{4}, \quad 1 \leq k \leq n .
$$


Using the representation of the integral as an iterated integral, one can show that

$$
\begin{array}{r}
\int_{U_{n}\left(n^{-1 / 2+\varepsilon}\right)} \phi_{j}^{4} e^{-a \vec{\theta}^{T} \hat{Q} \vec{\theta}-R_{k}(\vec{\theta})} d \vec{\theta} \leq c^{\prime} n^{2} \int_{U_{n}\left(n^{-1 / 2+\varepsilon}\right)} e^{-a \vec{\theta}^{T} \hat{Q} \vec{\theta}-R_{k}(\vec{\theta})} d \vec{\theta}+ \\
+O\left(\exp \left(-c n^{2 \varepsilon}\right)\right) \int_{\mathbb{R}^{n}} e^{-a \vec{\theta}^{T} \hat{Q} \vec{\theta}} d \vec{\theta}
\end{array}
$$

for some constants $c, c^{\prime}>0$, depending only on $a, b$ and $\gamma$.

Using (5.19), one can get that:

$$
\begin{array}{r}
\int_{U_{n}\left(n^{-1 / 2+\varepsilon}\right)} \theta_{j}^{4} e^{-a \vec{\theta}^{T} \hat{Q} \vec{\theta}-R_{k}(\vec{\theta})} d \vec{\theta} \leq \frac{c_{1}^{\prime}}{n^{2}} \int_{U_{n}\left(n^{-1 / 2+\varepsilon}\right)} e^{-a \vec{\theta}^{T} \hat{Q} \vec{\theta}-R_{k}(\vec{\theta})} d \vec{\theta}+ \\
+O\left(\exp \left(-c_{1} n^{2 \varepsilon}\right)\right) \int_{\mathbb{R}^{n}} e^{-a \vec{\theta}^{T} \hat{Q} \vec{\theta}} d \vec{\theta} ; \\
\int_{U_{n}\left(n^{-1 / 2+\varepsilon}\right)} e^{-a \vec{\theta}^{T} \hat{Q} \vec{\theta}-R_{k}(\vec{\theta})} d \vec{\theta} \geq\left(1+\frac{c_{2}^{\prime}}{n}\right) \int_{U_{n}\left(n^{-1 / 2+\varepsilon}\right)} e^{-a \vec{\theta}^{T} \hat{Q} \vec{\theta}-R_{k+1}(\vec{\theta})} d \vec{\theta}+ \\
+O\left(\exp \left(-c_{2} n^{2 \varepsilon}\right)\right) \int_{\mathbb{R}^{n}} e^{-a \vec{\theta}^{T} \hat{Q} \vec{\theta}} d \vec{\theta}
\end{array}
$$

for some constants $c_{1}, c_{1}^{\prime}, c_{2}, c_{2}^{\prime}>0$, depending only on $a, b$ and $\gamma$. Note that

$$
b \sum_{\left\{v_{j}, v_{k}\right\} \in E G} \Delta_{j k}^{4} \leq 8 b n \sum_{j=1}^{n} \theta_{j}^{4}=R_{1}(\vec{\theta}) .
$$

Using several times inequality (5.21) for $k=1,2, \ldots, n-1$ and combining with (5.22), (5.17), we obtain (5.16).

Proof of Lemma 4.1. Let

$$
F(\vec{\theta})=\exp \left(-a \vec{\theta}^{T} \hat{Q} \vec{\theta}-b \sum_{\left\{v_{j}, v_{k}\right\} \in E G} \Delta_{j k}^{4}\right) .
$$

Note that for $\theta \in L$,

$$
F(\vec{\theta})=\exp \left(-a \sum_{\left\{v_{j}, v_{k}\right\} \in E G} \Delta_{j k}^{2}-b \sum_{\left\{v_{j}, v_{k}\right\} \in E G} \Delta_{j k}^{4}\right) .
$$

Since the integrand of (4.7) is invariant under uniform translation of all the $\theta_{j}$ 's $\bmod \pi$ as well as $V_{0}$ are mapped into itself, we can fix $\bar{\theta}=0$ and multiply it by the ratio of its range $\pi$ to the length $n^{-1 / 2}$ of the vector $\frac{1}{n}[1,1, \ldots, 1]^{T}$. Thus 
we get that

$$
\begin{array}{r}
\int_{V_{0}} \exp \left(-a \sum_{\left\{v_{j}, v_{k}\right\} \in E G} \Delta_{j k}^{2}-b \sum_{\left\{v_{j}, v_{k}\right\} \in E G} \Delta_{j k}^{4}\right) d \vec{\theta}= \\
=\pi n^{1 / 2} \int_{L \cap U_{n}\left(n^{-1 / 2+\varepsilon}\right)} F(\vec{\theta}) d L .
\end{array}
$$

In a similar way as in (5.15) we find that

$$
\begin{gathered}
\int_{L \cap U_{n}\left(n^{-1 / 2+\varepsilon}\right)} F(\vec{\theta}) d L=\int_{P(\vec{\theta}) \in U_{n}\left(n^{-1 / 2+\varepsilon}\right)} F(\vec{\theta}) d \vec{\theta} / \int_{-\infty}^{+\infty} e^{-a n x^{2}} d x= \\
=\left(\frac{\pi}{a n}\right)^{-1 / 2} \int_{P(\vec{\theta}) \in U_{n}\left(n^{-1 / 2+\varepsilon}\right)} F(\vec{\theta}) d \vec{\theta} .
\end{gathered}
$$

Using (5.11) and (5.14), we get that

$$
\int_{P(\vec{\theta}) \in U_{n}\left(n^{-1 / 2+\varepsilon}\right)} F(\vec{\theta}) d \vec{\theta}=\int_{U_{n}\left(n^{-1 / 2+\varepsilon}\right)} F(\vec{\theta}) d \vec{\theta}+O\left(\exp \left(-c n^{2 \varepsilon}\right)\right) \int_{\mathbb{R}^{n}} e^{-a \vec{\theta}^{T} \hat{Q} \vec{\theta}} .
$$

Combining (5.16), (5.25), (5.26) and (5.27), we obtain (4.7).

\section{Final remarks}

Combining (4.2), (4.5), (4.6), 4.32), (5.25), (5.26), (5.27), we find that:

Proposition 6.1. Let the assumtions of Theorem 2.1 hold. Then

$$
E O(G)=\left(1+O\left(n^{-1+6 \varepsilon}\right)\right) 2^{|E G|-1 / 2} \pi^{-n+1 / 2} n \text { Int },
$$

where

$$
\begin{array}{r}
\text { Int }=\int_{U_{n}\left(n^{-1 / 2+\varepsilon)}\right.} \exp \left(-\frac{1}{2} \vec{\theta}^{T} \hat{Q} \vec{\theta}-\frac{1}{12} \sum_{\left\{v_{j}, v_{k}\right\} \in E G} \Delta_{j k}^{4}\right) d \vec{\theta}, \\
\hat{Q}=Q+J, \quad \Delta_{j k}=\theta_{j}-\theta_{k},
\end{array}
$$

where $Q=Q(G)$ denotes the Laplcian matrix and $J$ is the matrix with every entry 1 .

Integral (6.2) can be evaluated more precisely for specific classes of graphs in order to get asymptotic formulas for $E O(G)$ similar to (1.1). For example, for even $n \rightarrow \infty$

$$
E O\left(K_{n, n}\right)=e^{-1} \frac{2^{n^{2}+n-\frac{1}{2}}}{\pi^{n-\frac{1}{2}} n^{n-1}}\left(1+O\left(n^{-1 / 2+\varepsilon}\right)\right)
$$

for any $\varepsilon>0$, where $K_{n, n}$ denotes the complete bipartite graph with $n$ vertices in each part. 


\section{Acknowledgements}

This work was carried out under the supervision of S.P. Tarasov and supported in part by RFBR grant no 11-01-00398a.

\section{Warning}

It is preprint of the paper "Asymptotic behavior of the number of Eulerian orientations of graphs" accepted for publication in "Mathematical Notes" in 2012, (C) by Pleiades Publishing, Ltd., see also http://www.maik.ru.

\section{References}

[1] M. Isaev, Asymptotic behaviour of the number of Eulerian circuits, Electronic Journal of Combinatorics, 2011, V.18(1), P.219, e-print arXiv: 1104.3046.

[2] M. Fiedler, Algebraic connectivity of graphs, Czech. Math. J. 23 (98) (1973), 298-305.

[3] G. Kirchhoff, Über die Auflösung der Gleichungen, auf welche man bei der Untersuchung der linearen Verteilung galvanischer Ströme geführt wird, Ann. Phys. Chem. 72 (1847), 497-508. Translated by J. B. O'Toole in I.R.E. Trans. Circuit Theory, CT-5 (1958) 4.

[4] M. Las Vergnas, Le polynôme de Martin d'un graphe eulérien, Ann. Discrete Math., 17 (1983), 397-411.

[5] M. Las Vergnas, An upper bound for the number of Eulerian orientations of a regular graph, Combinatorica, ISSN 1439-6912, Vol. 10 (1. 1990), p. $61-65$

[6] M. Mihail and P. Winkler, On the number of Eulerian orientations of a graph, Algorithmica 16 (1996), 402414.

[7] B. Mohar, The Laplacian spectrum of graphs, Graph Theory, Combinatorics, and Applications, Vol. 2, Ed. Y. Alavi, G. Chartrand, O. R. Oellermann, A. J. Schwenk, Wiley, 1991, pp. 871-898.

[8] B. D. McKay, R. W. Robinson, Asymptotic enumeration of eulerian circuits in the complete graph. Combinatorica, 7(4), December 1998.

[9] B. D. McKay, The asymptotic numbers of regular tournaments, eulerian digraphs and eulerian oriented graphs. Combinatorica 10 (1990), no. 4, $367-377$.

[10] A. Schrijver, Bounds on the number of Eulerian orientations, Combinatorica, 3 (1983), 375-380. 
M.I. Isaev

Centre de Mathématiques Appliquées, Ecole Polytechnique, 91128 Palaiseau, France

Moscow Institute of Physics and Technology,

141700 Dolgoprudny, Russia

e-mail: isaev.m.i@gmail.com 